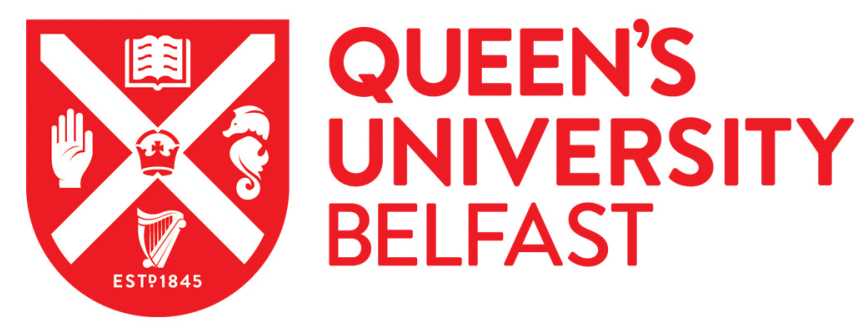

\title{
The Phosphorylation Mechanism of Mevalonate Diphosphate Decarboxylase, a QM/MM Study
}

McClory, J., Hui, C., Zhang, J., \& Huang, M. (2020). The Phosphorylation Mechanism of Mevalonate Diphosphate Decarboxylase, a QM/MM Study. Organic and Biomolecular Chemistry, 18(3), 518-529. https://doi.org/10.1039/C9OB02254F

Published in:

Organic and Biomolecular Chemistry

Document Version:

Peer reviewed version

Queen's University Belfast - Research Portal:

Link to publication record in Queen's University Belfast Research Portal

Publisher rights

(c) The Royal Society of Chemistry 2019. This work is made available online in accordance with the publisher's policies. Please refer to any applicable terms of use of the publisher.

\section{General rights}

Copyright for the publications made accessible via the Queen's University Belfast Research Portal is retained by the author(s) and / or other copyright owners and it is a condition of accessing these publications that users recognise and abide by the legal requirements associated with these rights.

Take down policy

The Research Portal is Queen's institutional repository that provides access to Queen's research output. Every effort has been made to ensure that content in the Research Portal does not infringe any person's rights, or applicable UK laws. If you discover content in the Research Portal that you believe breaches copyright or violates any law, please contact openaccess@qub.ac.uk. 


\title{
The Phosphorylation Mechanism of Mevalonate Diphosphate Decarboxylase, a QM/MM Study
}

\author{
James McClory ${ }^{\mathrm{a}}$, Chenggong Hui ${ }^{\mathrm{a}}$, Jian Zhang ${ }^{\mathrm{b}}$, Meilan Huang ${ }^{\mathrm{a}, *}$ \\ ${ }^{a}$ School of Chemistry and Chemical Engineering, Queen's University Belfast, David Keir \\ Building, Stranmillis Road, Belfast, BT9 5AG, Northern Ireland, United Kingdom \\ b Shanghai Jiaotong University, 280 Chongqing Road, Shanghai 200025, China
}

Corresponding author

*Meilan Huang

Phone: +442890974698

Fax: +442890976524

E-mail: m.huang@qub.ac.uk 


\section{ABSTRACT}

Mevalonate diphosphate decarboxylase (MDD) catalyses a crucial step of the mevalonate pathway via $\mathrm{Mg}^{2+}$-ATP dependent phosphorylation and decarboxylation reactions to ultimately produce isopentenyl diphosphate, the precursor of isoprenoids, which is essential to bacteria functions and offers ideal building blocks for the biosynthesis of isopentenols. However, the metal ion(s) in MDD has not been unambiguously resolved, which limits the understanding of the catalytic mechanism and the exploitation of the enzyme for the development of antibacterial therapies or the mevalonate metabolic pathway for the biosynthesise of biofuels. Here by analogizing the structurally related kinases and molecular dynamics simulations, we constructed a model of the MDD-substrate-ATP- $\mathrm{Mg}^{2+}$ complex and propose MDD requires two $\mathrm{Mg}^{2+}$ ions for maintaining a catalytically active conformation. Subsequent QM/MM studies indicate MDD catalyses the phosphorylation of its substrate mevalonate diphosphate (MVAPP) via a direct phosphorylation reaction, instead of the previously assumed catalytic base mechanism. The results here would shed light for the active conformation of MDD related enzymes and their catalytic mechanisms and therefore be useful for developing novel antimicrobial therapies or reconstructing mevalonate metabolic pathway for the biosynthesis of biofuels. 


\section{INTRODUCTION}

Mevalonate pathway is responsible for the biosynthesis of isopentenyl pyrophosphate (IPP) as a source of isoprenoids. Composed of more than 30,000 natural products, isoprenoids are closely related to various biological functions in many gram-positive bacteria, which has made the kinases involved in mevalonate pathway or glycolysis important drug targets for the development of antimicrobial agents; ${ }^{1}$ Moreover, isoprenoids are highly reactive energy-dense C5 building blocks for a wide range of products such as isopentenols. Therefore, these kinases are essential for the biosynthesis of isoprenoid-based biofuels and other valuable fine chemicals.

Mevalonate diphosphate decarboxylase (MDD; EC 4.1.1.33) is the last enzyme in the mevalonate (MVA) pathway and a key enzyme in engineered metabolic pathways for production of isobutene. Understanding the catalytic mechanism of the kinases involved in the MVA pathway would be helpful for exploiting the synthetic biology and pharmaceutical applications of these kinases. Recently, we reported the reaction mechanisms of MVA pathway kinases including isopentenyl phosphate kinase (IPK) ${ }^{2}$ and mevalonate kinase (MVK). ${ }^{3}$ However, the mechanism of MDD has not been elucidated due to the elusive location of magnesium ion(s).

Elucidation of the catalytic mechanism of MDD requires a complete complex structure of MDD in complex with ATP and $\mathrm{Mg}^{2+}$, which is essential for the catalytic function of the enzyme. However, so far, none of reported crystal structures of MDD contain $\mathrm{Mg}^{2+}$ in their catalytic sites. Here by analogizing with the $\mathrm{Mg}^{2+}$ ion coordination in pyruvate kinase, we suggest the catalytically competent structure of MDD requires two magnesium ions. Further, we disclosed the reaction mechanism of the phosphate transfer catalysed by MDD is via direct phosphorylation, instead of the conventionally deemed catalytic base mechanism. Such 
information is crucial for the exploitation of this kinase for rational drug design and its biotechnological application in sustainable production of biofuels.

\section{MATERIALS AND METHODS}

\subsection{Data Mining of Mg2+ coordination}

The positional probability of $\mathrm{Mg}^{2+}$ coordination in diverse proteins was investigated by a data mining study, in order to obtain the plausible location of $\mathrm{Mg}^{2+}$ in MDD. The crystal structures from the RCSB Protein Data Bank (PDB) were screened indiscriminately using $\mathrm{Mg}^{2+}$-ATP as the search criterion. The crystal structures of GHMP kinases such as PMK, MK and GALK all exhibit that a $\mathrm{Mg}^{2+}$ ion coordinated to at least one phosphate oxygen of the nucleotide $(\sim 2.5 \AA)$. Hence this was used as the screening criterion and a total of 100 protein crystal structures were chosen including a variety of proteins such as transferases, hydrolases, lyases and motor proteins. A statistical analysis was performed for the selected proteins to note the complete coordination sphere of magnesium of each protein and a complete list of the crystal structures used for the data mining is provided in the supplementary materials (Table S1).

\subsection{Protein Preparation}

The crystal structure of the D283W variant of Staphylococcus epidermidis MDD in complex with mevalonate diphosphate and ATP $\gamma \mathrm{S}$ (PDB code: 4DPW) was used to build the MDD complex model. ${ }^{4}$ The pose of the substrate in the wild-type MDD (PDB code: 4DPT) was adopted by superimposing two structures using Discovery Studio 4.0 (Accelrys USA). The nucleotide analogue ATP $\gamma \mathrm{S}$ was altered to the native nucleotide ATP by changing the sulphur atom of the $\gamma$-phosphate into oxygen and the alanine at position 283 was mutated back to the original aspartate of the WT enzyme. The magnesium ions and water ligands were manually placed in the structure in analogy to the Magnesium coordination in Saccharomyces cerevisiae pyruvate kinase (pdb code: $3 \mathrm{HQP}) .{ }^{5}$ All crystal waters were maintained. The protonation states 
were assigned by using ProPka and manual inspection. The pKa values of Asp283 and Lys188 were 0.4 and 14.7, respectively (at a $\mathrm{pH}$ of 7.0).

\subsection{MD simulations}

The AMBER 14 (University of California, USA) package was used for molecular dynamic simulations. ${ }^{6}$ All MD simulations were performed with the FF14SB forcefield. ${ }^{7}$ RESP charges of the MVAPP and ATP ligands was obtained by single point energy calculation using the Hartree-Fock (HF) method in conjunction with the 6-31G(d) basis set in Gaussian 09 package (Table S1). ${ }^{8}$ The GAFF forcefield ${ }^{9}$ was used for the ligands. The protein with 5139 protein atoms was soaked in a TIP3P water box within a minimum distance of $10 \AA$ to the surface of the protein. A total of 10,933 waters were added to the system. A total of $10 \mathrm{Na}^{+}$counter ions were added to maintain the protein neutral. Periodic boundary condition (PBC) was used in all MD simulations.

An initial minimization involving 1,250 steps of steepest descent minimization followed by 1,250 steps of conjugate gradient minimization was performed exclusively for the solute where the nucleotide, substrate and magnesium ions were restrained with a force constant of $50 \mathrm{kcal}$ $\mathrm{mol}^{-1} \AA^{-2}$. The optimization was terminated by checking the number of optimization step. After 2 rounds of optimization, the maximum force in any replicas was lower than $10 \mathrm{kcal} /(\mathrm{mol} * \AA)$. The restraint force was then removed and minimization was subsequently conducted on the entire protein system using 1,250 steps of steepest descent minimization followed by 1,250 steps of conjugate gradient minimization. The system was then heated gradually from $0 \mathrm{~K}$ to $300 \mathrm{~K}$ at a constant volume. Solute molecules were restrained using a harmonic potential of 50 kcal $\mathrm{mol}^{-1} \AA^{2}$ during the heating process. All hydrogen atoms were restrained using the SHAKE algorithm, where the relative geometrical tolerance 0.00001 was used for coordinate resetting. The restraint on the solute molecules was then removed and equilibration was 
performed using an NPT ensemble at $300 \mathrm{~K}$ and a constant pressure of 1 bar. An $8 \AA$ cut-off distance was set for the non-bonded van der Waals force and the Particle Mesh Ewald method was used to consider long-range electrostatic interactions. A 100ns production simulation was performed on the $2 \mathrm{Mg}^{2+}$-ATP-MDD-MVAPP complex using the NPT ensemble at $300 \mathrm{~K}$. Langevin thermostat with a collision frequency of $1 \mathrm{ps}^{-1}$ was used in the NPT heating and production of MD simulations. Pressure relaxation time was 2.0 ps for Barostat. Compressibility of the system was set as $44.6 \times 10^{-6}$ bar $^{-1}$ (the compressibility of water). In the heating and equilibrium process, time step of $1 \mathrm{fs}$ was used; a time step of $2 \mathrm{fs}$ was used in the production process of MD simulations.

Four replicas of the MD trajectory were performed for the complex. Root mean square deviation (RMSD) of the C $\alpha$ atoms was calculated (Figure S1) using the minimized crystal structure as the reference structure. Kmesans clustering algorithm was used for cluster analysis to obtain representative cluster structures from the MD trajectory, and the RMSD between all the $\mathrm{C} \alpha$ atoms was used as distance matrix.

\subsection{QM/MM Calculations}

The MD representative structure of MDD was optimized using a two-layer QM/MM method implemented in ONIOM of Gaussian 09 package (Gaussian, Inc.). ${ }^{8}$ The system was divided into two layers, namely the high layer (QM) and the low layer (MM). The high layer was optimized at the B3LYP/6-31+G (d) level of density functional theory while the low layer was optimized by the Amber Parm99 force field. Electronic embedding was used to integrate the partial charges of the two layers and a quadratically convergent Hartree-Fock (QC-SCF) method was applied. ${ }^{10}$ A reduced system size of $12 \AA$ from the active site was implemented with all atoms outside $6 \AA$ of the active site frozen using the protocol developed by Tao et al. ${ }^{11}$ MVAPP, two $\mathrm{Mg}^{2+}$ ions and the truncated nucleotide ATP with the methyl-triphosphate tail 
kept were included in the QM region, as well as the truncated Ser107, Ser141, Lys188, Ser192 and Asp283 with their side chains kept. The overall charge of the QM region was -4.

Potential energy scan (PES) was conducted for the QM/MM optimized geometry with a decrement of $0.1 \AA$ for the reaction coordinate, i.e. the distance between the $\gamma$-phosphate phosphorus of ATP and the C3-hydroxyl oxygen of MVAPP. The transition state (TS) structure obtained from the PES was optimized as TS and validated using frequency calculation. The energies of the stationary points were then corrected with the $6-31++G(d, p)$ basis set and the B3LYP functional (Table S2).

\section{RESULTS AND DISCUSSION}

\subsection{Indispensable $\mathrm{Mg}^{2+}$ ion in mevalonate pathway kinases}

MDD belongs to the GHMP kinase superfamily, ${ }^{12}$ a unique class of ATP-dependent enzymes named after galactokinase (GALK), ${ }^{13,14}$ homoserine kinase (HK), ${ }^{15}$ mevalonate kinase (MVK) ${ }^{16}$ and phosphomevalonate kinase (PMK). ${ }^{17}$ The kinases in the GHMP superfamily participate in several essential metabolic pathways, among which, the mevalonate pathway involved in the isoprenoid biosynthesis has attracted huge interest because of the disease and biotechnological relevance of isoprenoids. ${ }^{18,19,20,21,22}$

Mevalonate pathway utilizes the metabolite acetyl-CoA as a source of carbon to yield isopentenyl pyrophosphate (IPP), the precursors of isoprenoids. In the traditional mevalonate pathway, mevalonate is subjected to two successive phosphorylation reactions at the C5 position catalysed by MVK and PMK, respectively, while further phosphorylation occurs at the $\mathrm{C} 3$ position followed by decarboxylation catalysed by MDD to yield the product IPP (Scheme 1). An alternative pathway proceeds by phosphorylation at the C3 position of mevalonate catalysed by mevalonate-3-kinase (M3K) to give mevalonate-3-phosphate, which is then phosphorylated at the C5 position followed by decarboxylation catalysed by 
mevalonate-3,5-biphosphate decarboxylase (MBD) to give isopentenyl phosphate, which finally is subjected to further phosphorylation catalysed by isopentenyl phosphate kinase (IPK) to yield IPP (Figure 1).

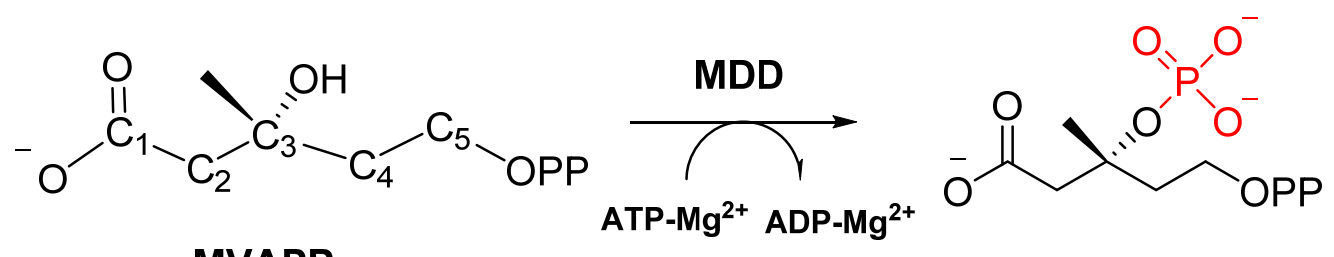

\section{MVAPP}

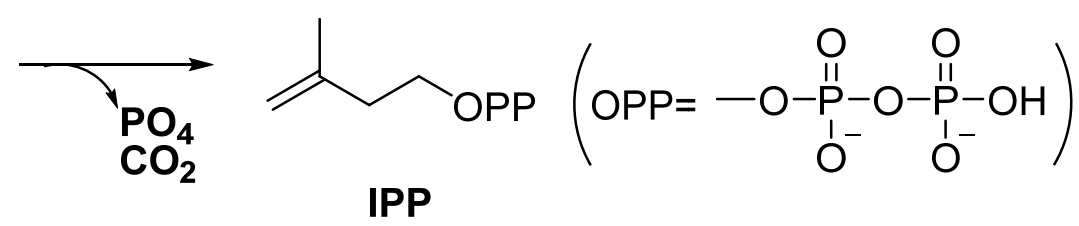

Scheme 1. MDD catalyses the phosphorylation of the 3-hydroxyl group of MVAPP, followed by a decarboxylation to yield isopentenyl pyrophosphate (IPP)

Alternative mevalonate pathways or reconstructed mevalonate pathway have been reported. It was found single amino acid mutation converted MDD into a M3K in the alternate mevalonate pathway ${ }^{23}$. Further, a bifurcate mevalonate pathway was discovered, and 5-phosphate decarboxylase (MPD) was found to catalyse the decarboxylation mevalonate 5-phosphate to give isopentenyl phosphate, which is then phosphorylated to give IPP. ${ }^{24}$ Both MDD and MPD possess the high dual specificity toward MVAP and MVAPP, which facilitates the reconstruction of mevalonate pathway. Understanding the catalytic mechanism of MDD would shed light for constructing alternative mevalonate metabolic pathways via MDD related enzymes for the terpene biosynthesis. 


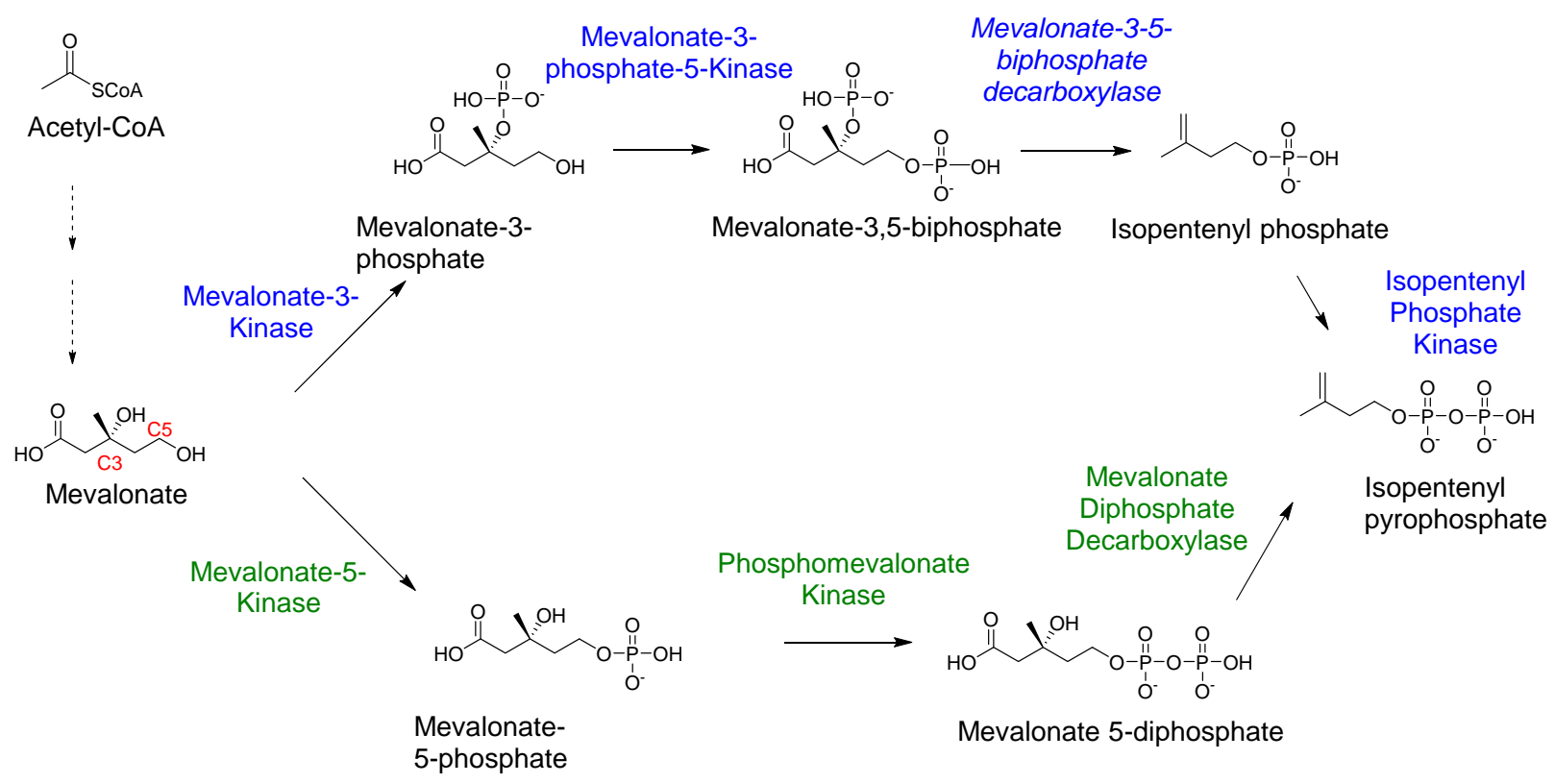

Figure 1: Enzymes involved in the mevalonate pathway for the production of isopentenyl pyrophosphate (IPP). The enzymes shown in green are found in Eukarya while the kinases shown in blue are found in Archae.

Except for IPK that belongs to AAK family, all other enzymes in the mevalonate pathway belong to GHMP kinase family. In the structures of GHMP kinases, a $\mathrm{Mg}^{2+}$ ion is often coordinated to the phosphate groups of the nucleotide ATP and in some instances also coordinated to the substrate, i.e. $\mathrm{Mg}^{2+}$ is coordinated to both ATP and the substrate in PMK. ${ }^{25}$ The active sites of the mevalonate pathway enzymes, particularly, MDD, are heavily condensed in negative charge and require positively charged residues and metal ions to stabilize ATP cofactor and substrates. A kinetic study on chicken liver MDD indicated the kinase requires a metal ion, particularly $\mathrm{Mg}^{2+}$ to catalyse the phosphorylation and subsequent decarboxylation of MVAPP. ${ }^{26}$ Despite the important role of $\mathrm{Mg}^{2+}$, the location of $\mathrm{Mg}^{2+}$ has not been resolved in any of the crystal structures of MDD deposited in the Protein Databank (PDB), e.g. MDDSE ${ }^{4}$ and MDDEF. Even in crystal structures of the homologous MPD ${ }^{24}$ or the highly related mevalonate 3-kinase, ${ }^{23}$ no $\mathrm{Mg}^{2+}$ has been defined. This suggests it is difficult to resolve the 
location of $\mathrm{Mg}^{2+}$ ions in these enzymes and the reported crystal structures of these enzymes to date may represent inactive conformations of the enzymes for the catalytic reaction.

The lack of $\mathrm{Mg}^{2+}$ and the unavailability of the bioactive conformations of the MDD has largely limited the understanding of its catalytic mechanism and the exploitation of the MDD related enzymes for the development of new antimicrobial therapies or their biotechnological applications in constructing novel metabolic pathways for the production of isoprenoids.

\subsection{The catalytically competent complex of MDD containing two $\mathrm{Mg}^{2+}$ ions}

\subsubsection{Construction of MDD with one $\mathrm{Mg}^{2+}$ ion}

In the crystal structures of mevalonate pathway kinases reported so far, $\mathrm{Mg}^{2+}$ was only resolved in one GHMP kinase MVK and an AAK family kinase IPK. ${ }^{27}$ In order to decide the $\mathrm{Mg}^{2+}$ location in MDD, a statistical data mining study in conjunction with molecular docking and molecular dynamics (MD) was carried out. The crystal structures of 100 proteins that contain $\mathrm{Mg}^{2+} / \mathrm{ATP}$ in the protein databank (PDB) were used for the statistical analysis.

It was found that the conformations with $\mathrm{Mg}^{2+}$ coordinated to the $\beta / \gamma$ phosphate oxygen atoms of ATP are dominant, constituting $40 \%$ of the structures in the dataset (Figure S2, Table S3). In $32 \%$ of the crystal structures, $\mathrm{Mg}^{2+}$ is coordinated to the $\alpha / \beta / \gamma$ phosphates of the nucleotide, constituting the second most common conformation. Furthermore, the water or residues that are coordinated to the divalent metal ion in each protein structure were analyzed and it was found that the metal ion is coordinated to at least one water ligand in $68 \%$ of the crystal structures. Among those, the $\mathrm{Mg}^{2+}$ is most commonly found to be coordinated with two water, representing $24 \%$ of the structures with coordinating water. Coordination with three waters is also commonly found, representing $21 \%$ of the structures. In addition, $\mathrm{Mg}^{2+}$ may form coordination to a catalytic residue such as aspartic acid. For example, coordination with aspartic acid constitutes $24 \%$ of all the analyzed structures. These findings were used as the guidance to build the initial $\mathrm{Mg}^{2+}$ complex models of MDD in complex with the substrate.

The metal ion was first positioned to coordinate to the $\beta / \gamma$-phosphate of ATP, representing the most dominant magnesium coordination based on the data mining. The remaining octahedral 
coordination of $\mathrm{Mg}^{2+}$ is composed of the C3-hydroxyl of the substrate MVAPP and three water ligands, mimicing the three waters coordinated to the magnesium ion in the crystal structure of Streptococcus pneumonia PMK (PDB Code: $3 \mathrm{GON}$ ). ${ }^{25}$ The MD simulated structure of MDD complex showed that the diphosphate tail of MVAPP is tethered by the surrounding residues Ser141, Ser139 and Tyr18 at the active site via H-bond interactions (Figure S3A). Asp283, which was previously suggested to function as a catalytic base, ${ }^{28}$ formed a $\mathrm{H}$-bond with one of the coordinated water ligands. The hydroxyl side chain of Ser192 is H-bonded with Lys188, which in turn formed an ionic interaction with the $\gamma$-phosphate of ATP. Alternative MDD complex models with one magnesium were built based on the data mining analysis, however, MD simulations of these strucures led to abnormal coordination of the metal ion, for example, two oxygen atoms on the gamma phosphate of ATP formed abnormal coordination with $\mathrm{Mg}$. Alternatively, either ATP or substrate moved too far apart during time evolution such that it would no longer be feasible for the phosphorylation reaction to happen.

The $\mathrm{Mg}^{2+}$ ion was also made coordinated to the $\gamma$-phosphate of ATP and three water ligands, in analogy to PMK. The remaining coordination of magnesium was saturated by 3-C hydroxyl. MD simulation of such complex resulted in a conformation where Ser192 is H-bonded to the $\gamma$-phosphate of ATP and Ser141 formed a hydrogen bond with a coordinating water (Figure S3B). It should be noted that during the MD simulation Asp283 approached the catalytic centre to replace one of the coordinating water ligands so as to coordinate to the magnesium ion. The model appeared to be catalytically feasible with one appropriate $\mathrm{Mg}$ coordination and relative locations of the substrates. However, subsequent QM/MM scans didn't locate a transition state corresponding to a phosphoryl transfer, indicating the structure was not catalytically feasible.

\subsubsection{Coordination of the two $\mathrm{Mg}^{2+}$ ions in MDD}

Kinases transfer a phosphate group from a phosphate donor to an acceptor. While most of kinases use ATP as the phosphate donor, a few kinases use atypical donors. e.g. pyruvate kinase (PyK) uses phosphenolpyruvate (PEP) as the phosphate donor. Functionally related kinases may have similar structural domains. ${ }^{29}$ Although MDD and PyK belong to two distinct 
enzyme families, PyK also catalyses the phosphorylation of the substrate followed by a decarboxylation reaction. In addition, their substrates MVAPP and phosphenolpyruvate (PEP) are highly similar, both being highly negative with phosphate group and carboxylate group (Figure 3, Figure S4). Interestingly, comparison of the structures of MDD and PyK disclosed that the two enzymes exhibit striking similarity in the active site configuration. Noting that two magnesium ions are required for catalysis by $\mathrm{PyK},{ }^{5}$ we postulated that MDD may also need two $\mathrm{Mg}^{2+}$ ions for substrate binding and its catalytic activity, and therefore built a MDD model with two $\mathrm{Mg}^{2+}$ ions configured analogous to PyK.

In the crystal structure of PyK, one Magnesium ion is coordinated to the $\alpha-, \beta-, \gamma$-phosphates of ATP and one water ligand. This represents the phosphorylated product structure where the phosphate group of the PEP substrate has been transferred to a second substrate ADP (Figure S4), indicating the phosphate of the substrate PEP to be transferred is also coordinated to the magnesium ion. Concomitant coordination of $\mathrm{Mg}^{2+}$ with the $\alpha-, \beta-, \gamma$-phosphates of ATP was also observed in a homologous GHMP kinase human GALK. Hence, the first magnesium ion (denoted as MG1) in the MDD complex was positioned to be coordinated to the $\alpha-, \beta-, \gamma-$ phosphate of ATP as well as the $\alpha$-phosphate of the substrate to be transferred (Figure 3 ). On the Walker P-loop ${ }^{30}$ of the GHMP kinases, a highly conserved serine residue (Ser142 in human GALK and Ser146 in rat MVK) is observed which is coordinated to $\mathrm{Mg}^{2}$ (Figure 2). Therefore, in the MDD- $\mathrm{Mg}^{2+}$-ATP-MVAPP complex, the hexa-coordination of MG1 was saturated by the hydroxyl side chain of the conserved Ser107 residue along with one water ligand.

Both PyK and MDD catalyse a substrate with a carboxylate group (Figure S4). In PyK (Figure 3B), the second magnesium is coordinated to the $\beta-, \gamma-$ phosphate of the ATP (phosphate acceptor and donor, respectively) and the carboxylate group of the oxalate. Therefore, the second $\mathrm{Mg}^{2+}$ ion in the MDD complex model, denoted as MG2, was also placed at similar configuration to be coordinated with the C3-hydroxyl group and the $\gamma$-phosphate of ATP 
(phosphate acceptor and donor, respectively) as well as the carboxylate of the substrate MVAPP. This configuration is favourable for stabilizing the associated negative charge of the carboxylate moiety and is also analogous to the magnesium coordination in a GHMP kinase PMK ${ }^{25}$ that was proposed to be pivotal in aligning the substrate and nucleotide for in-line phosphate transfer. ${ }^{31}$ In addition, similar to PMK, three water ligands were added to saturate the remaining coordination.

When building the complex structures of MDD containing two metal ions (and containing one met ion), we actually placed the metal in many different positions in regard to ATP, such as at the $\beta / \gamma$ phosphates of ATP and exclusively at the $\gamma$-phosphate of ATP. However, after MD simulations these models became unreasonable, whereby ATP and mevalonate diphosphate moved too far apart for a phosphoryl transfer reaction to occur. Hence, these models were not investigated further.

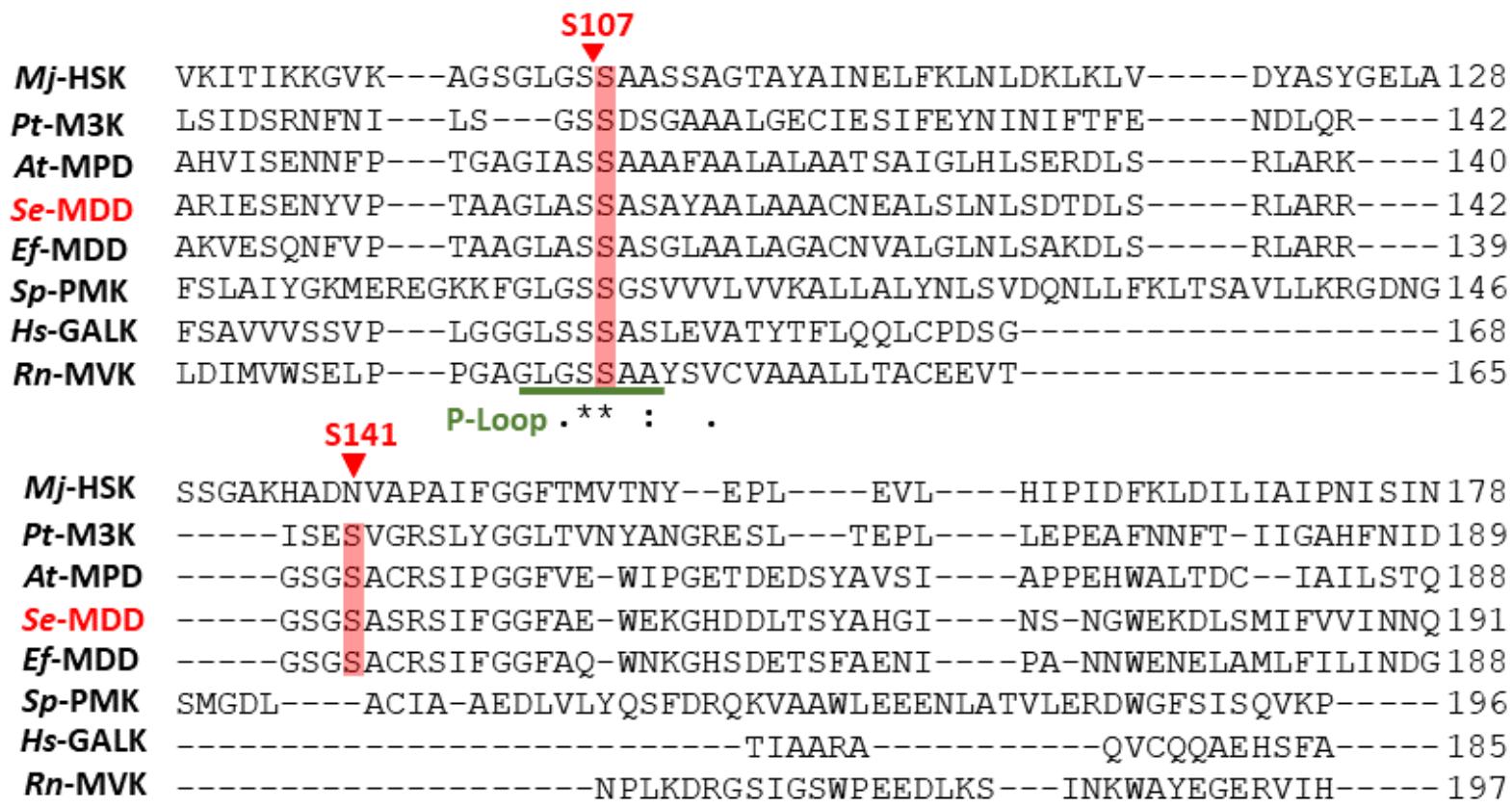




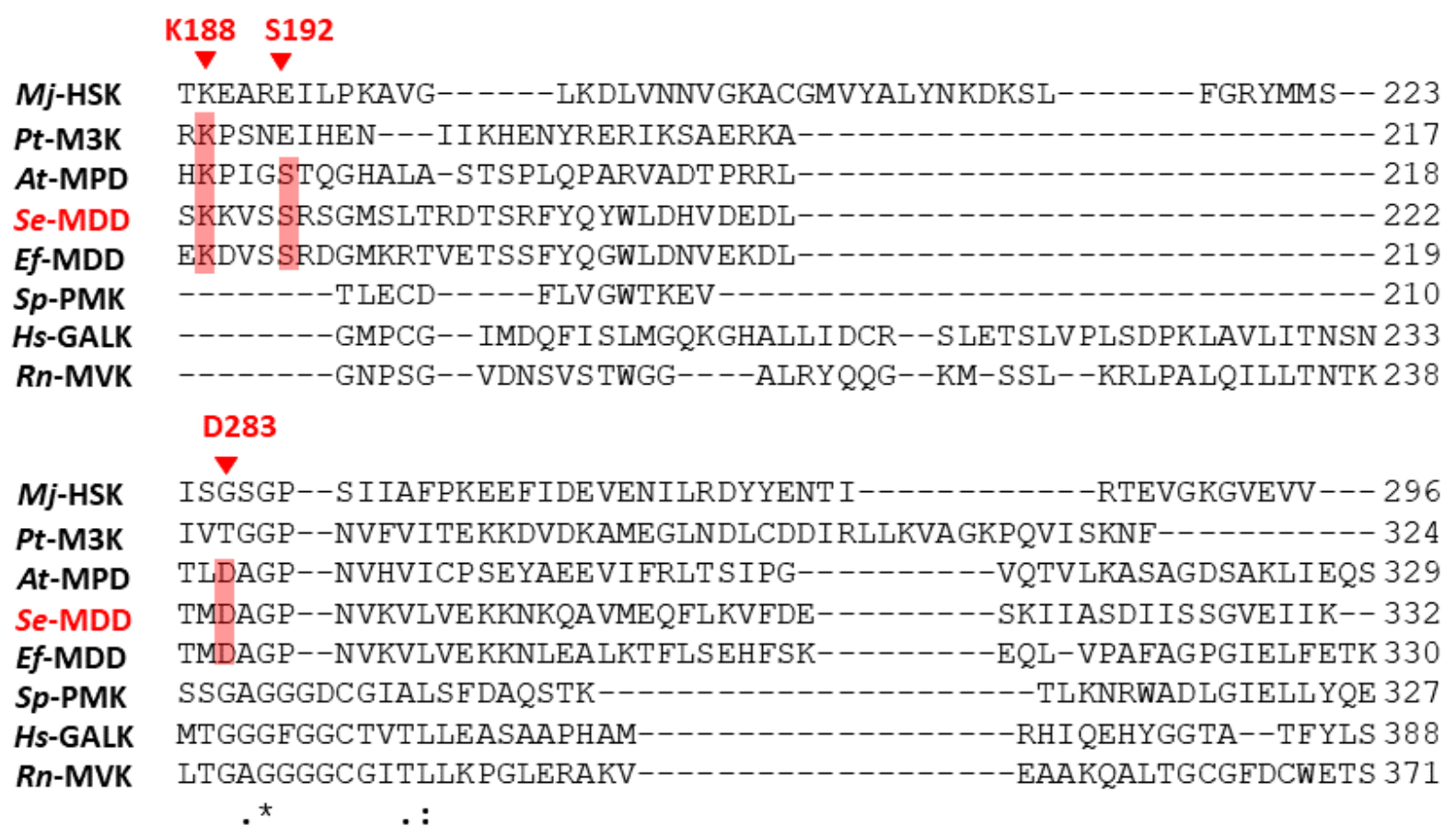

Figure 2. Sequence alignment of GHMP kinases using the Multiple Sequence alignment by Omega alignment tool provided by the European Bioinformatics Institute. The abbreviations are as follows: Methanocaldococcus jannaschii HSK (Mj-HSK: Q58504.2); Picrophilus torridus M3K (Pt-M3K: AAT43941); Anaerolinea thermophila MPD (At-MPD); Staphylococcus epidermidis MDD (Se-MDD: AAG02436.1); Enterococcus faecalis MDD (EfMDD: AAO80711.1); Streptococcus pneumonia PMK (Sp-PMK: AAK99144.1); Homo sapiens GALK (Hs-GALK: P51570.1); Rattus norvegicus MVK (Rn-MVK: P17256.1). The residues Ser107, Ser141, Lys188, Ser192 and Asp283 of Se-MDD that were included in the QM region of the QM/MM calculations are highlighted in red bars. Ser107 on the P-loop (underlined by green line) in Se-MDD is highly conserved among the GHMP kinase, corresponding to Ser146 in Rn-MVK and Ser142 in Hs-GALK.

The catalytic residue Lys188 is located on a highly dynamic loop connecting $\beta$-sheet 9 and helix 5 (residues 182-192). It is exposed when MDD is bound with its substrate MVAPP (PDB: 4DU7) but the loop (185-192) where Lys188 is located is not resolved in the enzyme complexed with an inhibitor and ATP (PDB: 4DPT). In the D283A variant (PDB: 4DPW), 
Lys 188 orientates favourably towards the phosphate tail of ATP. ${ }^{4}$ Compared with the WT enzyme, the D283A mutation causes a $120^{\circ}$ rotation of the carboxylate and C3-hydroxyl of the substrate MVAPP, as a result, the relative orientation of carboxylate in relation to Arg144 is unfavourable for the decarboxylation reaction to occur. In order to produce a more catalytically competent complex structure, the pose of the substrate was taken from the crystal structure of the WT enzyme and a complex model was obtained where the C3-hydroxyl of MVAPP orientates toward the $\gamma$-phosphate of ATP, and therefore favourable for a phosphorylation reaction (Figure 3).

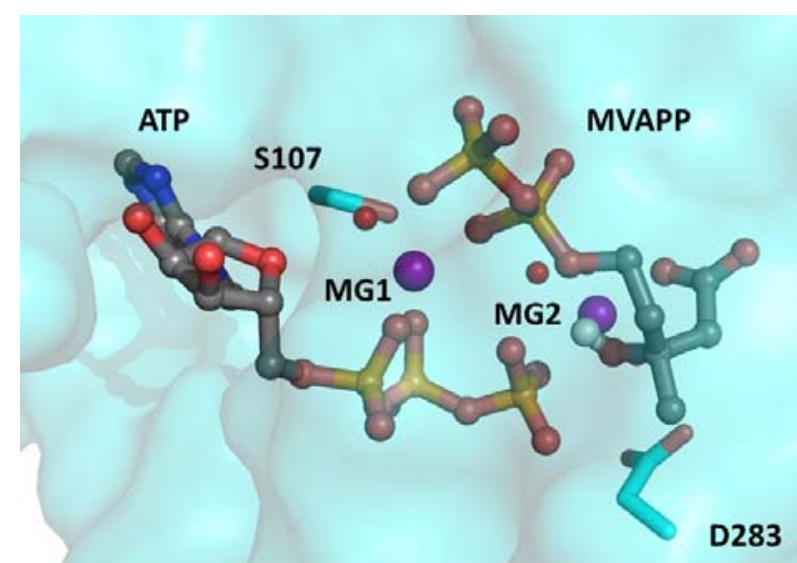

(a)

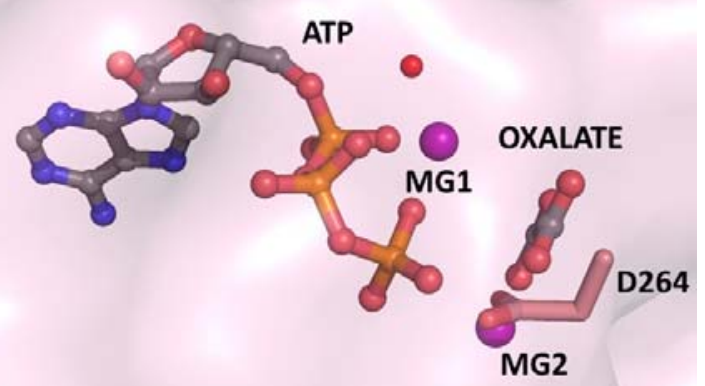

(b)

Figure 3. PyK was used as the template to build the starting model for MDD- $2 \mathrm{Mg}^{2+}-\mathrm{ATP}-$ MVAPP model. (a) 100-ns MD simulated structure for MDD coordinated with MVAPP, two magnesium ions and ATP (obtained from replica 1). (b) The crystal structure of PyK in complex with oxalate, two magnesium ions (PDB: 3HQP) and the phosphorylated product ATP.

A 100-ns MD simulation was run for the complex and the initially built catalytic site configuration retained stable throughout the simulation (Figure 3). The RMSD analysis shows that the system is well equilibrated during the MD simulations (Figure S1). In the crystal 
structure of WT MDD, Lys188 is exposed. Strikingly, it turned inward toward the $\beta$-, and $\gamma-$ phosphate of ATP, when the nucleotide is present (Figure 4). This significant conformational change in turn caused the closing of the loop Pro99-Ala105 (Figure 4 \& Figure S5), which is above the flexible triphosphate tail of the nucleotide, so as to rigidify it for the subsequent phosphoryl transfer. Similar to the crystal structure, ${ }^{4}$ the $\beta$-phosphate of the substrate MVAPP in the MDD-ATP-Mg ${ }^{2+}$-MVAPP complex forms a H-bond with Ser139 and an ionic interaction with Lys21 and Arg193 (Figure 4), while the $\alpha$-phosphate of MVAPP forms H-bond interactions with Ser107 and Ser141. Compared with the crystal structure Lys72 further approached the adenine part of the nucleotide, locking it in the active site for the reaction to happen.

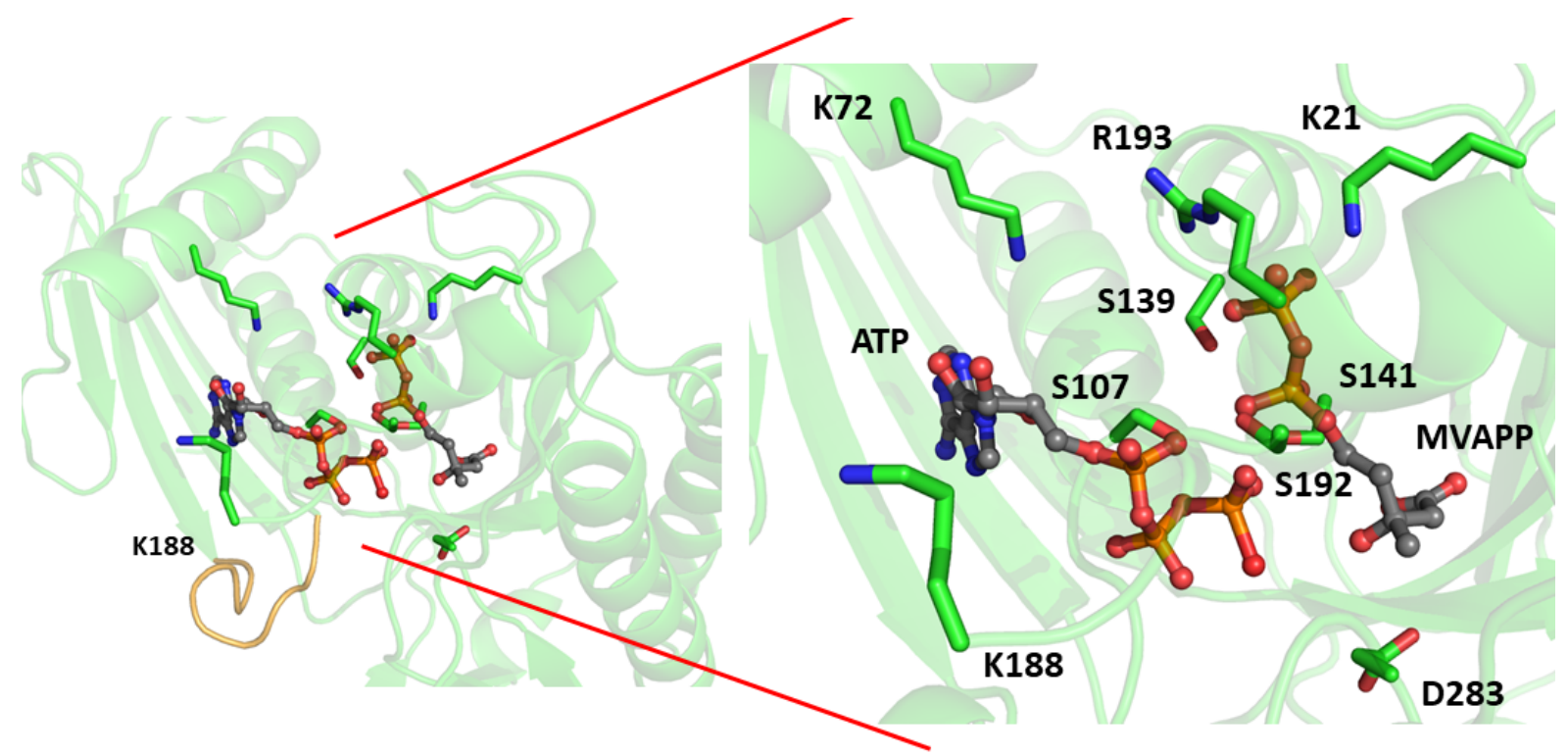

(a) 


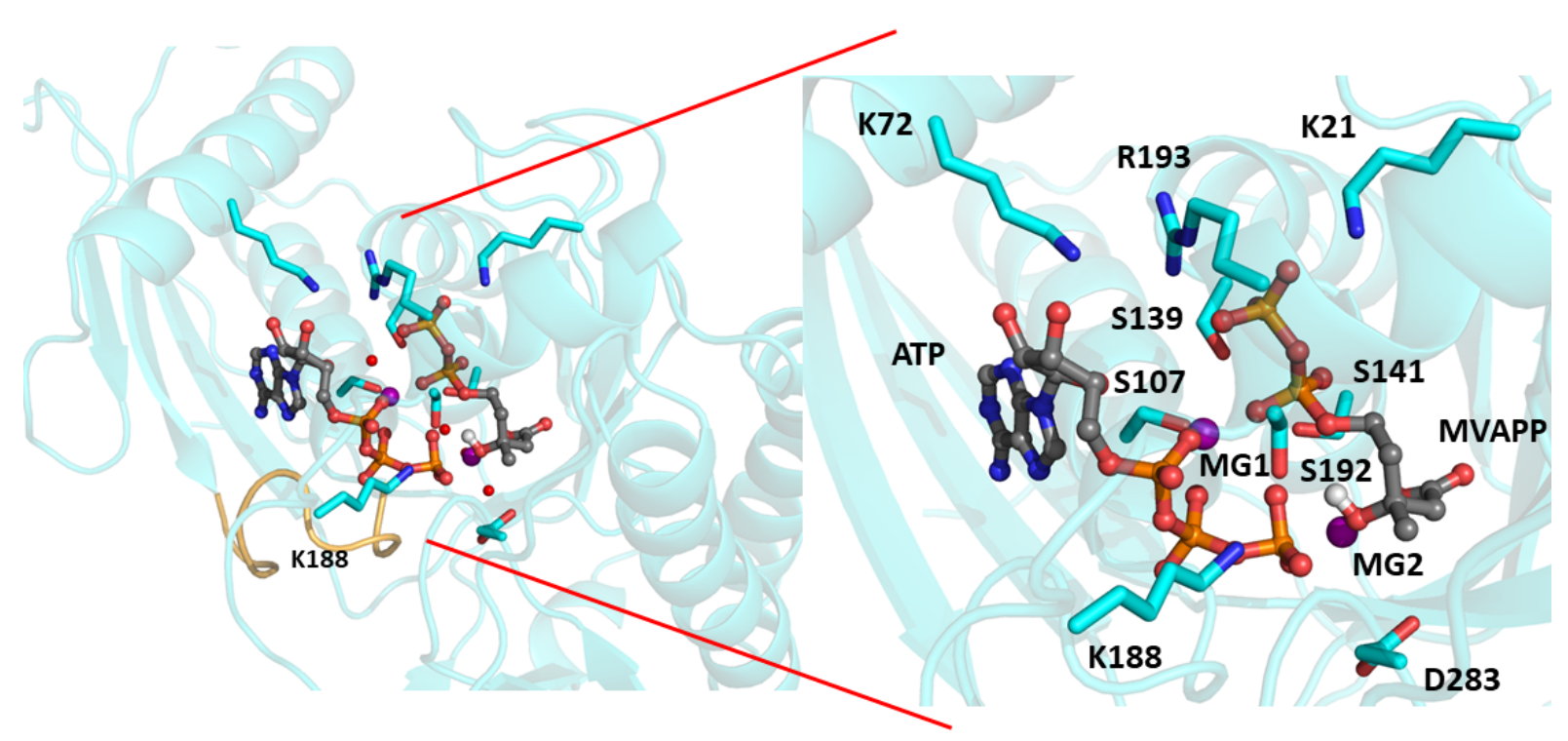

(b)

Figure 4. (a) The crystal structure of WT MDD complexed with substrate MVAPP (PDB: 4DU7). ATP was missing in the crystal and was taken from the crystal structure of the D283A mutant for illustration. (b) MD simulated structure of MDD-ATP- $\mathrm{Mg}^{2+}-\mathrm{MVAPP}$.

The catalytically favourable structure of MDD in complex with its substrate MVAPP, ATP and two magnesium ions not only would provide the basis for understanding the catalytic mechanism of MDD and its homologous kinases such as MPD and M3K, but also enable the investigation of the active conformations of these MDD homologous enzymes and their substrate scope for constructing alternative mevalonate metabolic pathways for isoprenoid biosynthesis.

\subsection{MDD catalyses the phosphorylation via a direct phosphorylation mechanism}

The reaction process catalysed by MDD is complicated including phosphorylation, dephosphorylation and decarboxylation. In addition, reaction needs to overcome a barrier associated with the conformational change of the phosphorylated intermediate 3P-MVAPP prior to decarboxylation. ${ }^{23}$ MDD catalyses the phosphorylation of MVAPP and subsequent decarboxylation. The phosphorylation gives 3-phosphate mevalonate 5-diphosphate (3P- 
MVAPP) was originally assumed to decompose spontaneously due to the instability of the sterically hindered tertiary phosphate. ${ }^{32-33}$ However, a stepwise reaction mechanism was then proposed based on the observed stable analogous intermediates. ${ }^{34,35}$ Furthermore, the intermediate in D281V and D281T mutants of Sulfolobus solfataricus MDD ${ }^{23}$ were suggested to inhibit the decarboxylation step rather than phosphate transfer, indicating that MDD catalyses the phosphorylation and decarboxylation via consecutive steps rather than a spontaneous mechanism. ${ }^{36}$

The phosphorylation catalysed by MDD was previously assumed to be facilitated by a negatively charged aspartate residue that was proposed as a catalytic base. ${ }^{37}$ However, the variant with a D281N mutation still exhibited some enzymatic activity, ${ }^{23}$ which is in conflict with its presumed role of catalytic base. A putative MDD homologue from $P$. torridus was found to be actually a M3K, another key kinase in the mevalonate pathway that catalyses the phosphorylation of MVA to mevalonate 3-phosphate. ${ }^{38,39}$ Superimposition of MDD and its highly related homologous kinase Thermoplasma acidophilum M3K ${ }^{38}$ shows there is a threonine instead of aspartate in $\mathrm{M} 3 \mathrm{~K}$, and mutation of this residue showed no influence on the enzymatic activity, ${ }^{38}$ indicating the aspartate in MDD may be crucial for the decarboxylation step rather than phosphorylation. Furthermore, it should be noted that in the catalytically active conformation of the MDD complex obtained from MD simulations, the previously deemed Asp283 was not in the close proximity to the substrate C3-hydroxyl to abstract its hydrogen. Therefore, it's not possible for it to function as a catalytic base and forfeit further study of catalytic base mechanism.

Because of the complexity of the enzyme and the uncertainty of key catalytic residues involved in the reaction process, it's difficult to resolve the controversy around the reaction mechanism by experimental techniques. Computational modelling particularly combined QM/MM methods provide a valuable atomic-level insight into the reactions of enzymes. ${ }^{40}$ In order to 
understand how MDD catalyses phosphate transfer from ATP to the substrate MVAPP, reaction mechanism studies were performed for the MDD-2 $\mathrm{Mg}^{2+}$-ATP-MVAPP complex using QM/MM method. The QM region is composed of MVAPP, the two magnesium ions, ATP and the water ligands that are coordinated to the metal ions. previous kinetic study showed that mutation of S107 that is coordinated to one of the metal ions caused a 42,000 decrease in $k_{\text {cat }},{ }^{41}$ therefore Ser107 was also included in the high layer.

Previous QM/MM studies on GHMP kinases MVK, ${ }^{3} \mathrm{PMK}^{31}$ and GALK ${ }^{42}$ revealed that a positively charged residue at the $\beta$-, $\gamma$ - bridging oxygen of ATP would neutralise the negative charge which accumulates upon phosphoryl transfer. Therefore, the lysine residue in the proximity of ATP in MDD, Lys $188^{43}$ was included in the QM region. In addition, the catalytic residue Ser192 that is hydrogen-bonded with the $\gamma$-phosphate of ATP was included in the QM region, as well as Asp283 and Ser141 that are H-bonded with two water ligands coordinated with MG2. All these residues were truncated such that only the side chains of the residues were kept in the QM region. 


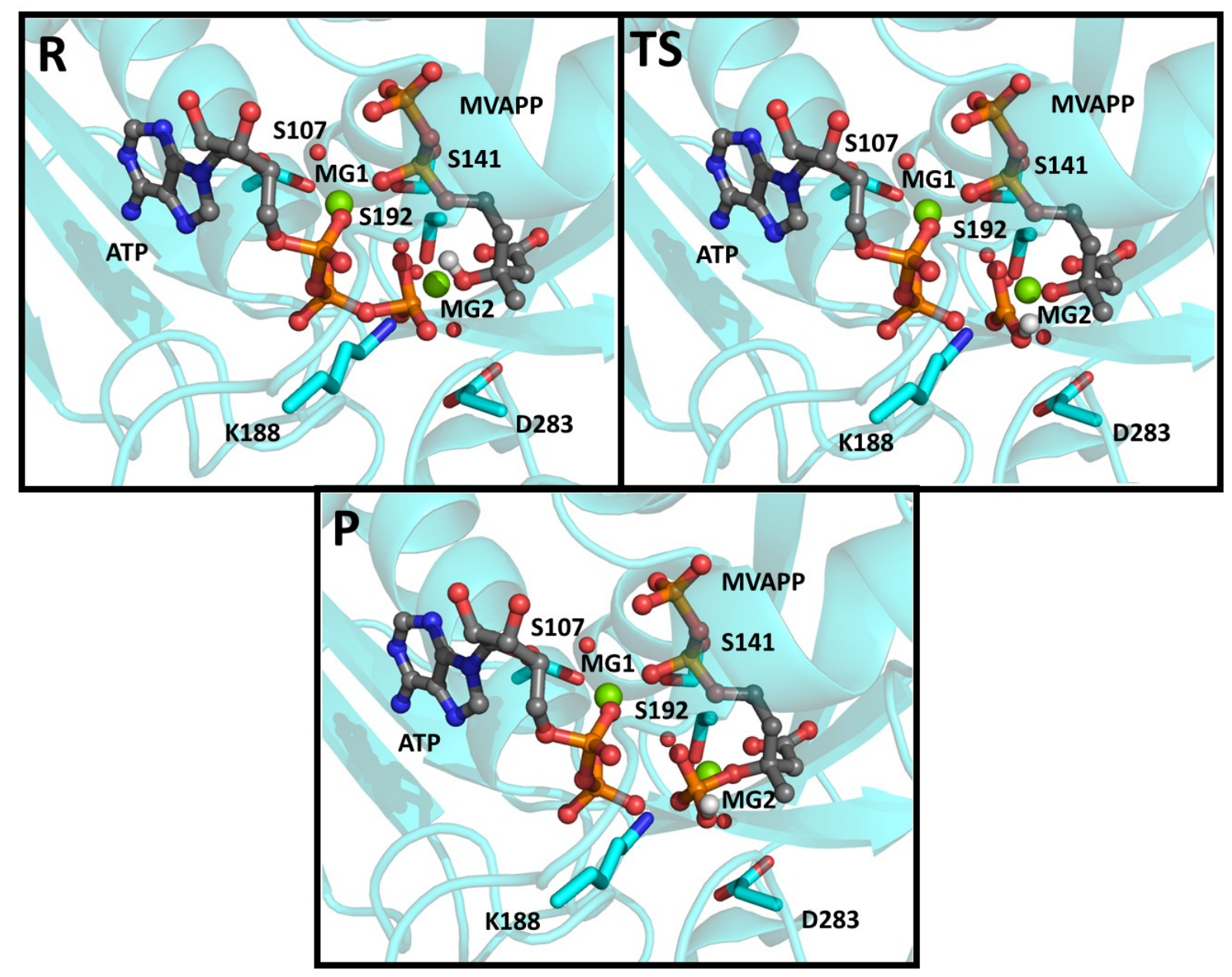

Figure 5. QM/MM optimized structures of the stationary points on the potential energy surface corresponding to reactant (R), transition state (TS) and product (P) of MDD. The calculations were conducted with the B3LYP/6-31+G(d) level of theory.

Potential energy surface scan was conducted with by scanning the distance between the C3hydroxyl $\mathrm{O}$ atom of MVAPP and the $\gamma$-phosphate P atom of ATP using QM/MM calculations. The results revealed that phosphorylation of the substrate MVAPP takes place via a concerted $\mathrm{S}_{\mathrm{N}} 2$ mechanism. A proton is abstracted by the terminal phosphate group of ATP while the C3hydroxyl group of MVAPP performs a nucleophilic attack on the $\gamma$-phosphate of ATP. As the $\gamma$-phosphate of ATP is pushed toward the C3-hydroxyl of MVAPP, the coordination of $\mathrm{Mg}^{2+}$ with ATP is changed from $\alpha-/ \beta-/ \gamma$ - phosphate in the initial model to $\alpha-/ \beta$ - phosphate, resulting in a pentacoordination of $\mathrm{Mg}^{2+}$, while the other magnesium ion remains in the 6-coordinate 
octahedral geometry (Figure 5). Such penta-coordinated $\mathrm{Mg}^{2+}$ has actually been reported previously in the phosphate transfer in the protein containing two- $\mathrm{Mg}^{2+}$ such as RhoA GDP. ${ }^{44}$ Throughout the reaction process, the catalytic residue Asp283 forms a hydrogen bond with a water ligand that is coordinated to MG1. Thus, we suggest that Asp283 plays an important role in organising the active site during the reaction, instead of functioning as a catalytic base as previously proposed. ${ }^{36}$ Further, our QM/MM studies on MDD demonstrate the phosphate transfer from the $\gamma$-phosphate of ATP to the C3-hydroxyl group of MVAPP occurs via a direct phosphorylation mechanism (Figure 6), instead of the previously deemed catalytic base mechanism. ${ }^{36}$

Previous QM/MM studies on two mevalonate pathway kinases $\mathrm{PMK}^{31}$ and $\mathrm{MVK}^{3}$ revealed that a positively charged residue (Lys101 and Arg241 in PMK and MVK, respectively) stabilizes the negative charge developed at the $\beta, \gamma$-bridging oxygen of ATP upon phosphate transfer, indicating the lysine residue may be critical for the phosphorylation process of the kinases in the GHMP kinase family. From the QM/MM study, we find that Lys 188 in MDD also functions to stabilize the negative charge at the $\beta$-, $\gamma$-bridging oxygen of ATP, in addition, it forms a salt-bridge interaction with the $\alpha$-phosphate and $\gamma$-phosphate group of ATP during phosphate transfer. Thus, Lys188 plays crucial role in positioning of the nucleotide as well as stabilization the TS.

Ser192 forms a hydrogen bond with the $\gamma$ - phosphate group of ATP; in addition, it maintains an $\mathrm{H}$ bond with Lys 188 throughout the reaction process, indicating these two residues work in tandem to facilitate phosphate transfer. Mutating an invariant serine residue Ser121 in Saccharomyces cerevisiae MDD caused a 42,000 decrease in $k_{\mathrm{cat}}{ }^{41}$ and the corresponding Ser107 in Staphylococcus epidermidis MDD was proposed to position the phosphate tail of ATP. ${ }^{4}$ Our QM/MM studies show that Ser107 is coordinated to an $\mathrm{Mg}^{2+}$ ion; in addition, it 
forms an $\mathrm{H}$ bond with the $\alpha$-phosphate of MVAPP. Thus, Ser107 plays a pivotal role in the catalytic activity of MDD by coordinating with $\mathrm{Mg}^{2+}$ as well as positioning the substrate in the catalytic site.

Furthermore, we found an invariant residue Ser141 in Staphylococcus epidermidis MDD also forms a hydrogen bond with the $\alpha$-phosphate of MVAPP; in addition, it is hydrogen-bonded to a coordinating water ligand, which is in turn hydrogen-bonded to the $\gamma$-phosphate of ATP. Thus, Ser141 helps the positioning of ATP and MVAPP via the hydrogen interactions mediated by a coordinating water, facilitating the phosphate transfer. This is in agreement with the previous kinetic studies on yeast MDD in which mutating the invariant serine residue S155A resulted in 26-fold inflation in $K_{\mathrm{M}}$ for $\mathrm{Mg}$-ATP and a 16-fold increase in $K_{\mathrm{M}}$ for MVAPP. ${ }^{41}$

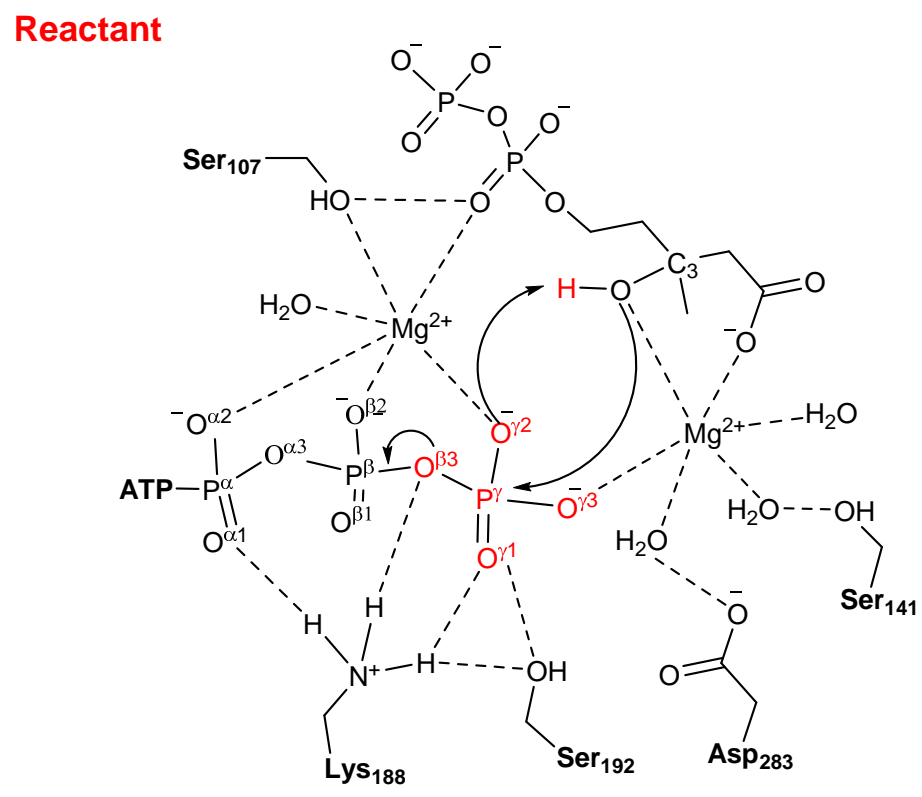


TS
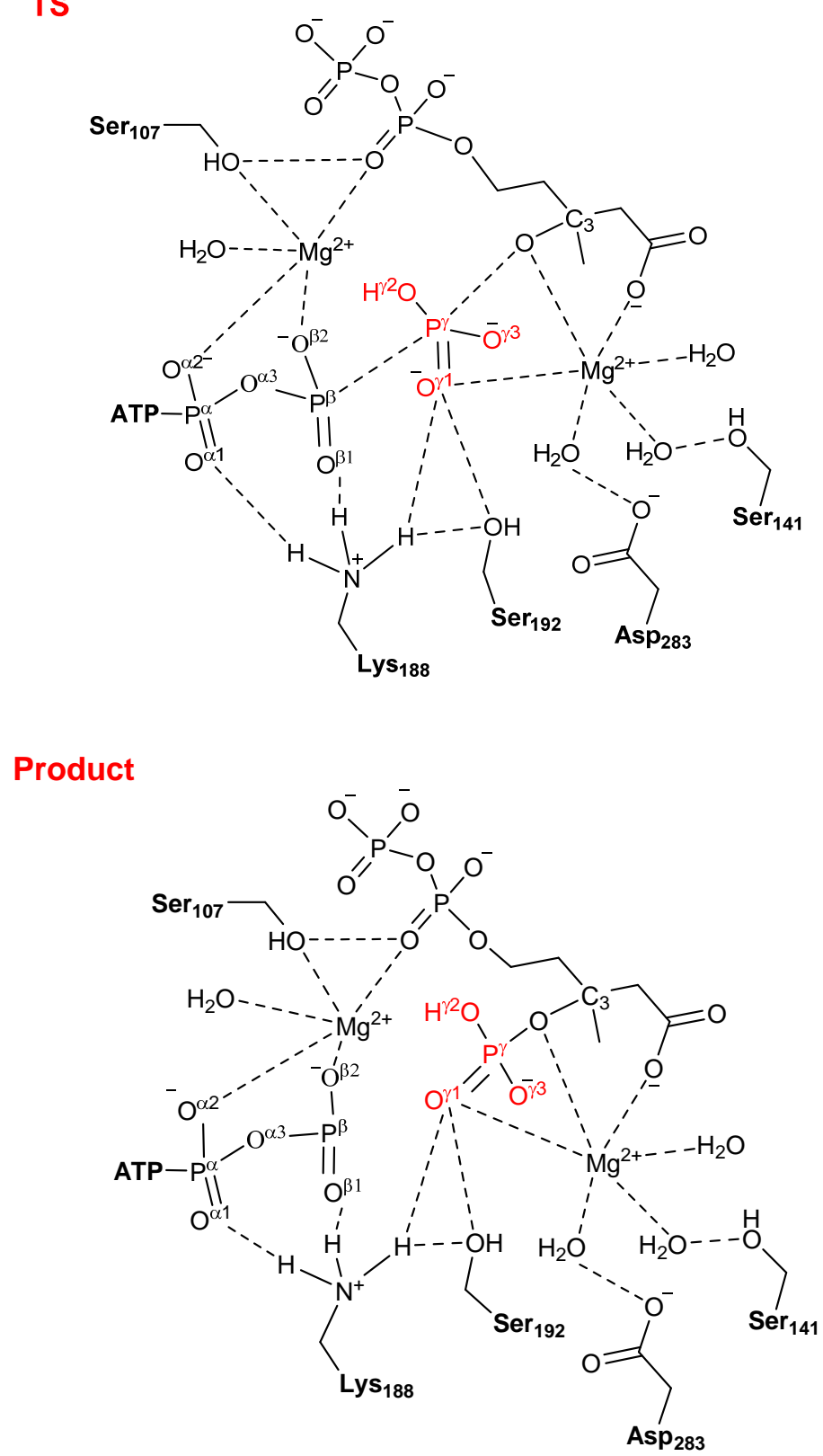

Figure 6. 2D sketch of the reaction mechanism of the phosphate transfer catalysed by MDD showing the reactant, transition state and product optimized using B3LYP/6-31+G(d) level of theory.

The transition state was located and validated by a unique imaginary frequency of $-191.99 \mathrm{~cm}^{-}$ ${ }^{1}$. In the TS structure, both the $r$ P $\gamma$-Oß3 distance $(d 1)$ and the $r$ O-P $\gamma$ distance $(d 2)$ are $2.04 \AA$. The TS structure exhibits a trigonal bipyramid structure with the transferring $\gamma$-phosphate group in planar configuration (Figure 5). The obtained metastable mevalonate-3-phosphate-5- 
diphosphate $(5.3 \mathrm{kcal} / \mathrm{mol}$ higher than mevalonate-5-diphosphate) subsequently undergoes spontaneous decarboxylation with the concomitant release of inorganic phosphate and carbon dioxide, yielding IPP. ${ }^{39}$ The decarboxylation is energetically favourable, no matter whether it proceeds with a two-step mechanism or a concerted mechanism via a tertiary carbocation, and therefore making the phosphorylation the rate-determining step in the reactions catalysed by MDD. The estimated Gibbs free energy activation barrier of MDD is $17.4 \mathrm{kcal} / \mathrm{mol}$ (Figure 7), in good agreement with the experimental reaction barrier of $15.5 \mathrm{kcal} / \mathrm{mol}$ for Staphylococcus epidermis MDD. ${ }^{33}$ The effect of enlarging the basis set was considered by single point energy calculations for the stationary point using a larger basis set $6-311++G(d, p)$ with the B3LYP functional. Little difference was observed in the calculated reaction energies compared to those calculated using 6-31+G(d) basis set (Table S2).

Pauling's formula ${ }^{45} D(n)=D(l)-0.6 \log n$ was used to measure the associative or dissociative nature of the TS, where the term $\mathrm{D}(l)$ is the average length of a P-O bond $(1.73 \AA)$ and $\mathrm{D}(n)$ is calculated by average of $r$ O-P $\gamma$ and $r$ P $\gamma$-Oß3 in the transition state structure. Based on the QM/MM optimized transition state geometry, the estimated fractional bond number $(n)$ is 0.31 , indicating a dissociative character of $69 \%$. The dissociative feature of MDD concurs with that was observed for other GHMP kinase enzymes PMK ${ }^{31}$ and MVK, ${ }^{3}$ which exhibited dissociative feature of $82 \%$ and $39 \%$, respectively. 


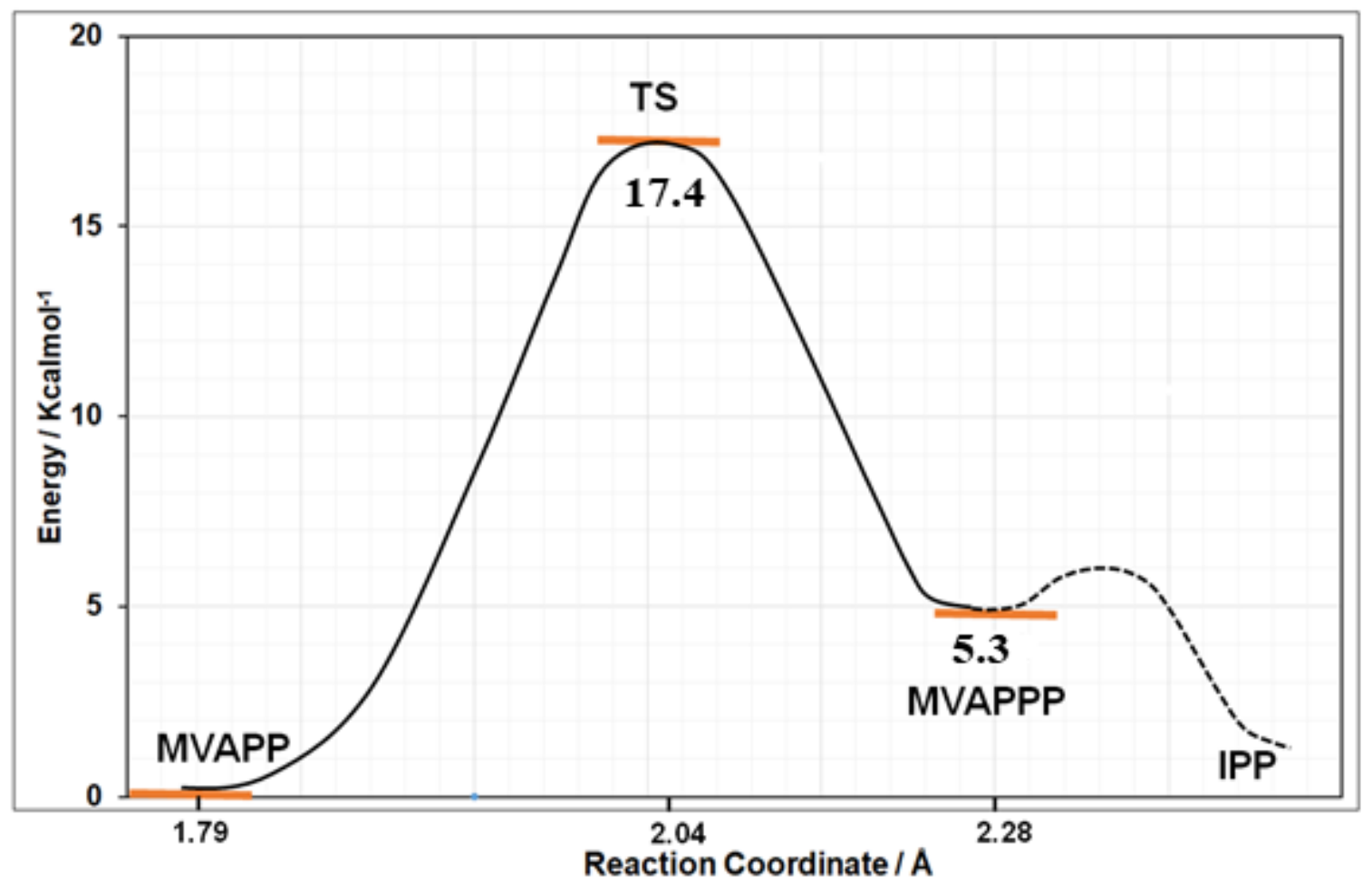

Figure 7: Reaction profile for phosphorylation of MVAPP in MDD (solid line). The Gibbs free energies were obtained by optimizations using QM/MM with the QM region calculated at the B3LYP/6-31+G(d) level. The subsequent decarboxylation is illustrated by a dashed curve.

\section{Conclusions}

The mevalonate pathway is pivotal in the Gram-positive bacterial pathogens such as enterococci, staphylococci, and streptococci, making the mevalonate pathway kinases attractive anti-bacterial drug targets. Moreover, the mevalonate pathway can be utilized for the biosynthesis of IPP, an important precursor for the production of isopentenol, therefore, it offers an attractive biosynthetic route for production of biofuels alternative to the everdepleting fossil fuels. MDD, a crucial enzyme in the mevalonate pathway, converts mevalonate diphosphate to produce IPP. So far, the crystal structure of MDD hasn't been well defined due to the lack of information on the divalent metal ions, which has greatly limited the exploitation of the kinase for development of antimicrobial therapies and biosynthesis of biofuels 
Here by analogizing with the $\mathrm{Mg}^{2+}$ ion coordination in pyruvate kinase, we propose that MDD requires two $\mathrm{Mg}^{2+}$ ions, to maintain a catalytically active structure of MDD. Through a QM/MM study we revealed the phosphorylation reaction catalysed by MDD is via direct phosphorylation, instead of the previously postulated catalytic base mechanism. In addition, similar to other mevalonate pathway kinases such as PMK and MVK, the transition state of the direct phosphorylation reaction exhibits a dissociative feature, although the latter kinases contain only one magnesium ion.

We found that Asp283 helps to organise the active site of MDD by interacting with the water ligands coordinated to a $\mathrm{Mg}^{2+}$ ion. In addition, The catalytic Lys188 helps to position the $\gamma$ phosphate of ATP throughout the reaction process and stabilizes the negative charge accumulating at the $\beta$-, $\gamma$-bridging oxygen of ATP upon phosphate transfer. Ser141 helps to position the substrate and ATP in line with phosphate transfer via the hydrogen bond interactions with a coordinating water. Ser192 assists in arranging the phosphate tail of ATP by interacting with the $\gamma$-phosphate of ATP and Lys188. Ser107 on the glycine-rich loop is coordinated to one of the magnesium ions and interacts with the phosphate groups of both ATP and the substrate MVAPP, indicating the invariant serine in the MDD plays a pivotal role in binding of the ATP-Mg as well as the substrate MVAPP.

Further, we elucidated the reaction mechanism of MDD, and found it catalysed the phosphorylation reaction via direct phosphorylation mechanism, with a negatively charged residue Asp283 in the catalytic site stabilizing a coordinating water via hydrogen bond instead of functioning as a catalytic base. This argues the commonly assumed catalytic base mechanism proposed for MDD.

In summary, despite the presence of a negatively charged residue present in the catalytic site, MDD catalyses the phosphorylation reaction via a direct phosphorylation mechanism, similar 
to other reported kinases in the mevalonate pathway including PMK, ${ }^{31} \mathrm{IPK}^{2}$ and MVK. ${ }^{3}$ This is in contrast to the commonly assumed catalytic base mechanism. In addition, similar to the other enzymes in this pathway, MDD was found to have a positively charged residue at the $\beta$ ,$\gamma$-bridging oxygen of ATP functioning to stabilize the accumulating negative charge upon phosphoryl transfer. MDD is the first mevalonate pathway enzyme and GHMP kinase that has been suggested to contain two $\mathrm{Mg}^{2+}$ ions. The complete structure of MDD in complex with mevalonate diphosphate, ATP and two $\mathrm{Mg}^{2+}$ ions presented here would provide valuable insights for developing novel biosynthetic route for the sustainable production of biofuels and would also be helpful for development of novel antibacterial therapies targeting mevalonate pathway kinases.

\section{SUPPLEMENTARY DATA}

Supplementary data is available on online.

\section{ACKNOWLEDGEMENTS}

J.M. acknowledges the financial support from the Department of Education and Learning (DEL), Northern Ireland. We are grateful for the computing resources from QUB high performance computing centre.

\section{REFERENCES}

1 E. Oldfield. Acc Chem Res. 2010;43(9):1216-26. doi: 10.1021/ar100026v.

2 J. McClory, D. J. Timson, W. Singh, J. Zhang and M. Huang, J Phys Chem B, 2017, 121, 11062-11071.: :10.1021/acs.jpcb.7b08770.

3 J. McClory, J. Lin, D. Timson, J. Zhang, M. Huang. Organic and Biomolecular Chemistry. 2019, 17, 2423-2431. doi: 10.1039/c8ob03197e. 
4 M. L. Barta, W. J. McWhorter, H. M. Miziorko and B. V. Geisbrecht, Biochemistry, 2012, 51, 5611-5621. dx.doi.org/10.1021/bi300591x.

5 H.P. Morgan, I.W. McNae, M.W. Nowicki, V. Hannaert, P.A. Michels, L.A. FothergillGilmore, M.D. Walkinshaw. J Biol Chem. 2010;285(17):12892-8. doi:

10.1074/jbc.M109.079905.

6 D. A. Case, V. Babin, J. T. Berryman, R. M. Betz, Q. Cai, D. S. Cerutti, T. E. Cheatham III, T. A. Darden, R. E. Duke, H. Gohlke, A. W. Goetz, S. Gusarov, N. Homeyer, P. Janowski, J. Kaus, I. Kolossváry, A. Kovalenko, T. S. Lee, S. LeGrand, T. Luchko, R. Luo, B. Madej, K. M. Merz, F. Paesani, D. R. Roe, A. Roitberg, C. Sagui, R. SalomonFerrer, G. Seabra, C. L. Simmerling, W. Smith, J. Swails, R. C. Walker, J. Wang, R. M. Wolf, X. Wu and P. A. Kollman, AMBER 14, University of California, San Francisco, 2014.

7 J. A. Maier, C. Martinez, K. Kasavajhala, L. Wickstrom, K. E. Hauser and C. Simmerling, J. Chem. Theory Comput., 2015, 11, 3696-3713.

8 M. J. Frisch, G. W. Trucks, H. B. Schlegel, G. E. Scuseria, M. A. Robb, J. R. Cheeseman, G. Scalmani, V. Barone, B. Mennucci, G. A. Petersson, H. Nakatsuji, M. Caricato, X. Li, H. P. Hratchian, A. F. Izmaylov, J. Bloino, G. Zheng, J. L. Sonnenberg, M. Hada, M. Ehara, K. Toyota, R. Fukuda, J. Hasegawa, M. Ishida, T. Nakajima, Y. Honda, O. Kitao, H. Nakai, T. Vreven, J. A. Montgomery, Jr., J. E. Peralta, F. Ogliaro, M. Bearpark, J. J. Heyd, E. Brothers, K. N. Kudin, V. N. Staroverov, R. Kobayashi, J. Normand, K. Raghavachari, A. Rendell, J. C. Burant, S. S. Iyengar, J. Tomasi, M. Cossi, N. Rega, J. M. Millam, M. Klene, J. E. Knox, J. B. Cross, V. Bakken, C. Adamo, J. Jaramillo, R. Gomperts, R. E. Stratmann, O. Yazyev, A. J. Austin, R. Cammi, C. Pomelli, J. W. Ochterski, R. L. Martin, K. Morokuma, V. G. Zakrzewski, G. A. Voth, P. Salvador, J. J. Dannenberg, S. Dapprich, A. D. Daniels, Ö. Farkas, J. B. 
Foresman, J. V. Ortiz, J. Cioslowski, and D. J. Fox, Gaussian 09, Revision D.01, Gaussian, Inc., Wallingford CT, 2009.

9 J. Wang, R. M. Wolf, J. W. Caldwell, P. A. Kollman and D. A. Case, J. Comput. Chem., 2004, 25, 1157-1174.

10 G. B. Bacskay, Chemical Physics, 1982, 65, 383-396. https://doi.org/10.1016/03010104(82)85211-7.

11 P. Tao and H. B. Schlegel. Journal of Computational Chemistry, 2010, 31, 2363-2369. DOI:10.1002/jcc.21524.

12 P. Bork, C. Sander and A. Valencia, Protein Sci., 1993, 2, 31-40. DOI:10.1002/pro.5560020104.

13 J. B. Thoden and H. M. Holden, J. Biol. Chem., 2003, 278, 33305-33311. DOI: 10.1074/jbc.R300025200.

14 H. M. Holden, J. B. Thoden, D. J. Timson and R. J. Reece, Cell. Mol. Life Sci., 2004, 61, 2471-2484. DOI: 10.1007/s00018-004-4160-6.

15 T. Zhou, M. Daugherty, N. V. Grishin, A. L. Osterman and H. Zhang, Structure, 2000, 8, 1247-1257. DOI:10.1016/s0969-2126(00)00533-5.

16 D. Yang, L. W. Shipman, C. A. Roessner, A. I. Scott and J. C. Sacchettini, J. Biol. Chem., 2002, 277, 9462-9467. DOI: 10.1074/jbc.M110787200.

17 M. J. Romanowski, J. B. Bonanno and S. K. Burley, Proteins, 2002, 47, 568-571. doi: 10.1074/jbc.M109.027425.

18 Y. Zheng, Q. Liu, L. Li, W. Qin, J. Yang, H. Zhang, X. Jiang, T. Cheng, W. Liu, X. Xu and M. Xian, Biotechnol Biofuels, 2013, 6, 57. doi: 10.1186/1754-6834-6-57. 
19 M. Li, R. Nian, M. Xian, H. Zhang. Appl Microbiol Biotechnol. 2018;102(18):7725-7738. doi: 10.1007/s00253-018-9200-5.

20 M. Li, F. Hou, T. Wu, X. Jiang, F. Li, H. Liu, M. Xian, H. Zhang. Nat Prod Rep. 2019. doi: 10.1039/c9np00016j.

21 M. Das, P. Patra, A. Ghosh. Renewable and Sustainable Energy Reviews, 2020, 1189: 109562. https://doi.org/10.1016/j.rser.2019.109562.

22 V. J. Martin, D. J. Pitera, S.T. Withers, J.D. Newman, J.D. Keasling. Nat Biotechnol. 2003;21(7):796-802. DOI:10.1038/nbt833.

23 K. Motoyama, H. Unno, A. Hattori, T. Takaoka, H. Ishikita, H. Kawaide, T. Yoshimura and H. Hemmi, J. Biol. Chem., 2017, 292, 2457-2469. doi: 10.1074/jbc.M116.752535.

24 S. T. Thomas, G. V. Louie, J. W. Lubin, V. Lundblad, J. P. Noel. ACS Chem Biol. 2019;14(8):1767-1779.

25 J. L. Andreassi, M. W. Vetting, P. W. Bilder, S. L. Roderick and T. S. Leyh, Biochemistry, 2009, 48, 6461-6468. doi: 10.1021/bi900537u.

26 A. M. Jabalquinto and E. Cardemil. Biochimica et Biophysica Acta., 1987, 916, 172-178.

27 M. F. Mabanglo, H. L. Schubert, M. Chen, C. P. Hill, C. D. Poulter, ACS Chem. Biol. 2010, 5, 517-527. doi: 10.1021/cb100032g.

28 N.E. Voynova, Z. Fu, K.P. Battaile, T.J. Herdendorf, J.J.P. Kim, H.M. Miziorko, Archives of Biochemistry and Biophysics, 2008, 480, 58-67.

29 J. Rauch, N. Volinsky, D. Romano, W. Kolch , Cell Commun Signal. 2011,9,23. DOI:10.1186/1478-811X-9-23.

30 J. E. Walker, M. Saraste, M. J. Runswick and N. J. Gay, EMBO J., 1982, 1, 945-951. 
31 M. Huang, K. Wei, X. Li, J. McClory, G. Hu, J.-W. Zou and D. Timson, J. Phys. Chem. B, 2016, 120, 10714-10722. DOI:10.1021/acs.jpcb.6b08480.

32 E. Byres, M. S. Alphey, T. K. Smith and W. N. Hunter, Journal of Molecular Biology, 2007, 371, 540-553. DOI: 10.1016/j.jmb.2007.05.094.

33 M. L. Barta, D. A. Skaff, W. J. McWhorter, T. J. Herdendorf, H. M. Miziorko and B. V. Geisbrecht, J. Biol. Chem., 2011, 286, 23900-23910. doi: 10.1074/jbc.M111.242016.

34 S. Dhe-Paganon, J. Magrath and R. H. Abeles, Biochemistry, 1994, 33, 13355-13362. DOI: 10.1021/bi00249a023.

35 J. M. Vinokur, T. P. Korman, Z. Cao, J. U. Bowie. Biochemistry. 2014;53(25):4161-8. doi: 10.1021/bi500566q.

36 J. M. Vinokur, T. P. Korman, M. R. Sawaya, M. Collazo, D. Cascio, J.U. Bowie. Protein Sci. 2015;24(2):212-20. doi: 10.1002/pro.2607.

37 D. Krepkiy and H. M. Miziorko, Protein Sci, 2004, 13, 1875-1881. DOI: $10.1110 /$ ps.04725204.

38 J. M. Vinokur, T. P. Korman, M. R. Sawaya, M. Collazo, D. Cascio and J. U. Bowie, Protein Sci., 2015, 24, 212-220. doi: 10.1002/pro.2607.

39 L. Rossoni, S. J. Hall, G. Eastham, P. Licence and G. Stephens, Appl. Environ. Microbiol., 2015, 81, 2625-2634. DOI: 10.1128/AEM.04033-14.

40 M. W. van der Kamp, A. J. Mulholland. Biochemistry. 2013; 52(16):2708-28. doi: $10.1021 / \mathrm{bi} 400215 \mathrm{w}$.

41 D. V. Krepkiy and H. M. Miziorko, Biochemistry, 2005, 44, 2671-2677. DOI: 10.1021/bi0484217. 
42 M. Huang, X. Li, J.-W. Zou and D. J. Timson, Biochemistry, 2013, 52, 4858-4868. dx.doi.org/10.1021/bi400228e.

43 Y. Qiu, J. Gao, F. Guo, Y. Qiao and D. Li, Bioorganic \& Medicinal Chemistry Letters, 2007, 17, 6164-6168. https://doi.org/10.1016/j.bmcl.2007.09.033.

44 D. L. Graham, P. N. Lowe, G. W. Grime, M. Marsh, K. Rittinger, S. J. Smerdon, S. J. Gamblin and J. F. Eccleston, Chemistry \& Biology, 2002, 9, 375-381. DOI: 10.1016/S10745521(02)00112-6.

45 L. Pauling, "The Nature of the Chemical Bond," $3^{\text {rd }}$ ed. Cornell University Press, Ithaca, N.Y., 1960, p255.

Table of Contents 

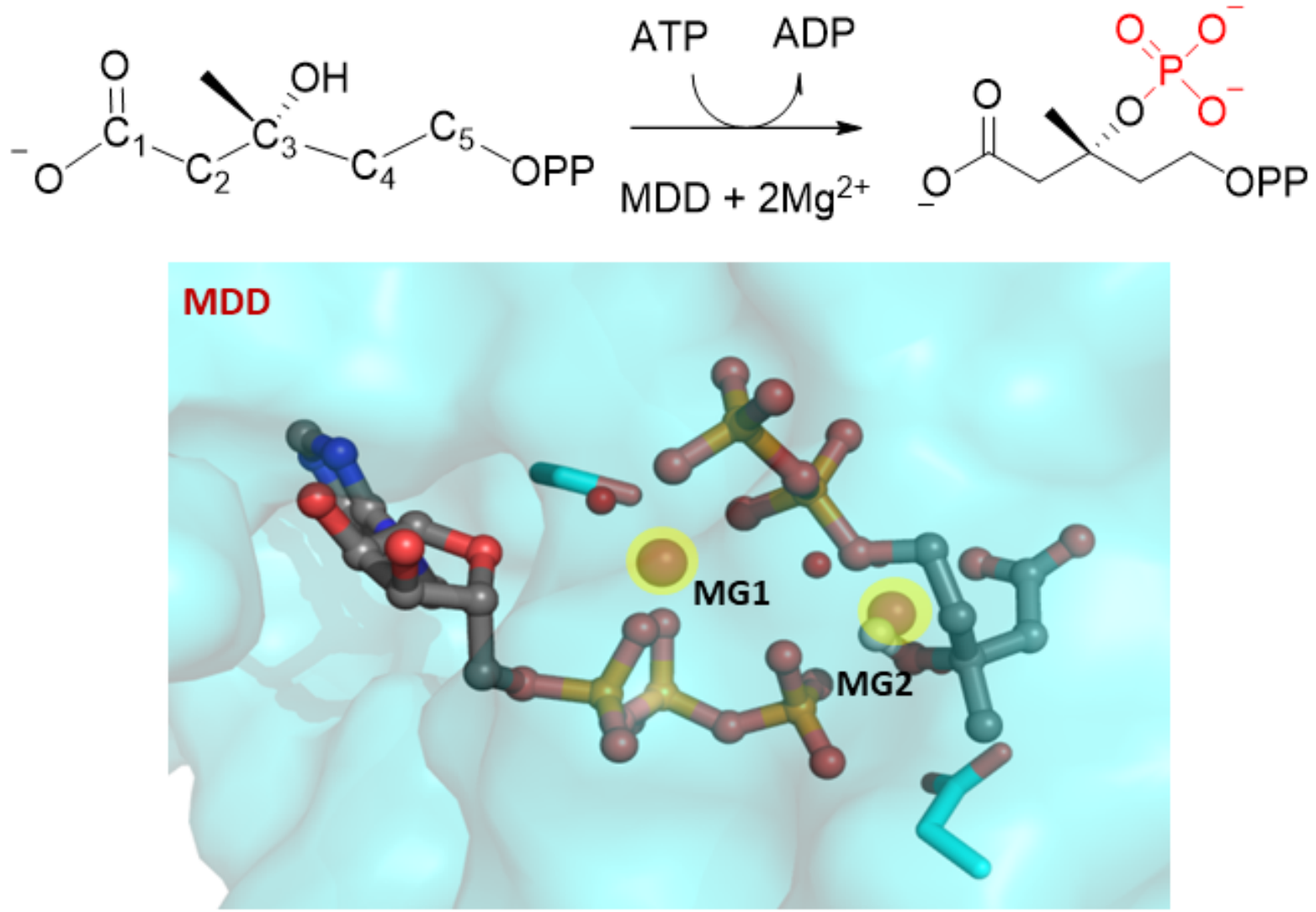

Combined MD and QM/MM studies suggested that Mevalonate Diphosphate Decarboxylase requires two magnesium ions for the phosphorylation reaction. 